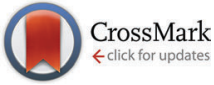

Cite this: Chem. Commun., 2016, 52,92

Received 26th August 2015, Accepted 21st October 2015

DOI: $10.1039 / c 5 c c 07185 b$

www.rsc.org/chemcomm

\section{The effect of branching in a semiconducting polymer on the efficiency of organic photovoltaic cells†}

\author{
Gaël H. L. Heintges, ${ }^{a}$ Jacobus J. van Franeker, ${ }^{\text {ab }}$ Martijn M. Wienk ${ }^{\text {ac }}$ and \\ René A. J. Janssen ${ }^{\star a c}$
}

\begin{abstract}
The impact of branching in a diketopyrrolopyrrole polymer on the performance of polymer-fullerene photovoltaic cells is investigated. Compared to the linear polymer, the branched polymer affords a more finely dispersed fibrillar network in the photoactive layer and as a result a large enhancement of the photocurrent and power conversion efficiency.
\end{abstract}

Polymer-fullerene photovoltaic cells have recently broken the barrier of $10 \%$ power conversion efficiency. ${ }^{1-4}$ In these cells, the conjugated polymer is usually based on an alternating pattern of electron rich and electron poor moieties, offering control over the electronic and optical nature of the material. Among the more extensively investigated electron poor moieties is diketopyrrolopyrrole (DPP), a unit known to provide access to small bandgap polymers with high efficiencies ${ }^{5-7}$ and good ambipolar charge transport. ${ }^{8,9}$ Research efforts into these polymers have focused on the fine-tuning of the electronic and optical properties on the one hand, ${ }^{10}$ and the fine-tuning of the polymer-fullerene blend morphology on the other. The latter is done mostly via the influence of the solubilizing side-chains, ${ }^{11,12}$ or via the influence of the processing conditions. ${ }^{13-15}$ Almost all polymers that have been used in photovoltaic cells have a linear architecture, leaving the topic of alternative architectures largely unexplored. These alternative architectures are, however, synthetically accessible via the polycondensation reactions that are usually employed for conjugated polymers. These include brush-type structures, containing conjugated segments of defined length attached to the main chain, ${ }^{16,17}$ and defined or randomly branched structures. ${ }^{18-21}$ No reports on the photovoltaic properties of truly randomly branched polymers are available in literature to date. A possible

\footnotetext{
${ }^{a}$ Molecular Materials and Nanosystems \& Institute for Complex Molecular Systems, Eindhoven University of Technology, P. O. Box 513, 5600 MB Eindhoven,

The Netherlands. E-mail: r.a.j.janssen@tue.nl

${ }^{b}$ Dutch Polymer Institute (DPI), P. O. Box 902, 5600 AX Eindhoven,

The Netherlands

'Dutch Institute for Fundamental Energy Research, De Zaale 20,

5612 AJ Eindhoven, The Netherlands

$\dagger$ Electronic supplementary information (ESI) available. See DOI: 10.1039/c5cc07185b
}

advantage of this random architecture is the higher molecular weight that could be attained by increasing the average functionality of the monomers, as the influence of molecular weight on the blend morphology and the efficiency of polymer solar cells is often significant. ${ }^{6,15}$ Other effects potentially caused by the branching of stiff conjugated polymers remain unknown.

In this work, we present the effect of branching on DT-PDPPTPT, a polymer consisting of a DPP unit, substituted with relatively long 2-decyltetradecyl (DT) solubilizing side chains, alternating along the chain with a thiophene-phenylene-thiophene (TPT) conjugated segment (Scheme 1). DT-PDPPTPT was selected because of its good solubility and reasonable photovoltaic efficiency. ${ }^{5}$ It was found that introducing branching severely decreased solubility, even in the case of $1 \%$ of branching unit. However, this proved to have a surprisingly large effect on the photovoltaic efficiency and morphology.

DT-PDPPTPT was synthesized via the previously published procedure,${ }^{5}$ which was adapted by replacing a controlled amount of 1,4-bis(4,4,5,5-tetramethyl-1,3,2-dioxaborolan-2-yl)benzene by 1,3,5-tris(4,4,5,5-tetramethyl-1,3,2-dioxaborolan-2-yl)benzene to obtain the branched polymers. Two polymers were synthesized replacing 5\% and 1\% (5B-DT-PDPPTPT and 1B-DT-PDPPTPT respectively) of all bifunctional phenylene units by trifunctional units.

The polymer containing $5 \%$ of branching proved to be insoluble and will be excluded in the further discussion.
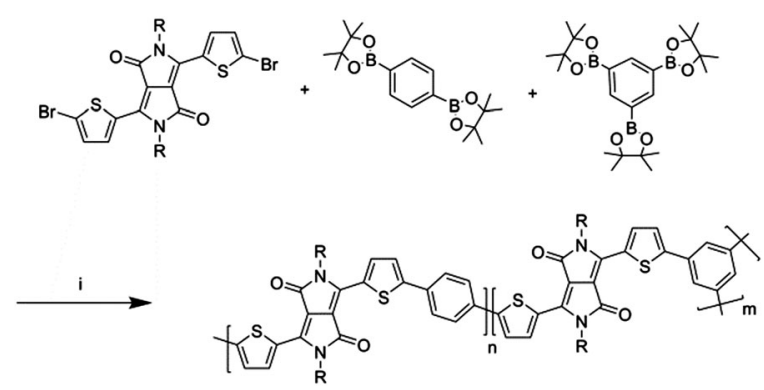

Scheme 1 Polymerization of branched DT-PDPPTPT, using a trifunctional phenylene core as branching unit. $R=2$-decyltetradecyl, (i) $\mathrm{Pd}_{2} \mathrm{dba}_{3}, \mathrm{PPh}_{3}$, $\mathrm{K}_{3} \mathrm{PO}_{4}$, toluene $/ \mathrm{H}_{2} \mathrm{O}, 115^{\circ} \mathrm{C} . \mathrm{m} /(m+n)=0,0.01$, or 0.05 . 


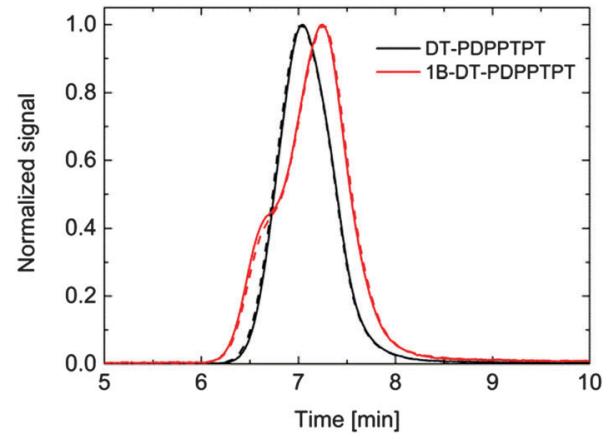

Fig. 1 GPC traces of DT-PDPPTPT and 1B-DT-PDPPTPT recorded in $\mathrm{ODCB}$ at $140{ }^{\circ} \mathrm{C}$. Two measurements are shown for each polymer, the second is indicated by the dotted line. The material corresponding to the shoulder around $6.3 \mathrm{~min}$. for 1B-DT-PDPPTPT showed a red-shifted absorption, and was assigned to aggregated 1B-DT-PDPPTPT.

The electronic and optical properties of the linear and branched polymers were expected and found to be very similar owing to their identical conjugated backbone. In both cyclic voltammetry and UV-vis-NIR absorption spectroscopy (Fig. S4 and S6, ESI $\dagger$ ) very similar properties were observed for both polymers. The molecular weight was estimated using gel permeation chromatography (GPC) analysis in hot ortho-dichlorobenzene $\left(o\right.$ DCB) at $140{ }^{\circ} \mathrm{C}$ (Fig. 1). A distinct shoulder was observed in the high molecular weight range for 1B-DT-PDPPTPT. This was attributed to aggregation as the UV-vis-NIR absorption profile of the material in this shoulder showed a red-shifted absorption profile.

Table 1 Photovoltaic performance of DT-PDPPTPT:[70]PCBM and 1BDT-PDPPTPT:[70]PCBM solar cells processed chloroform using DIO as co-solvent

\begin{tabular}{llllll}
\hline \multirow{2}{*}{ Polymer } & $\begin{array}{l}\text { DIO } \\
{[\mathrm{vol} \%]}\end{array}$ & $\begin{array}{l}J_{\mathrm{sc}}{ }^{a} \\
{\left[\mathrm{~mA} \mathrm{~cm}{ }^{-2}\right]}\end{array}$ & $V_{\mathrm{oc}}[\mathrm{V}]$ & $\mathrm{FF}$ & $\mathrm{PCE}^{b}[\%]$ \\
\hline DT-PDPPTPT & 3 & $7.75(7.43)$ & $0.80(0.80)$ & $0.68(0.67)$ & $4.2(4.0)$ \\
DT-PDPPTPT & 6 & $8.65(8.25)$ & $0.79(0.80)$ & $0.64(0.65)$ & $4.4(4.3)$ \\
1B-DT-PDPPTPT & 3 & $12.5(12.4)$ & $0.79(0.79)$ & $0.64(0.63)$ & $6.3(6.2)$ \\
1B-DT-PDPPTPT & 6 & $11.7(11.5)$ & $0.79(0.79)$ & $0.63(0.63)$ & $5.9(5.7)$
\end{tabular}

${ }^{a} J_{\mathrm{sc}}$ is obtained by integrating the EQE-measurement with the AM1.5G spectrum. ${ }^{b}$ Values between brackets indicate the average over 5 devices.
This indicates that 1B-DT-PDPPTPT has a strong tendency to aggregate compared to DT-PDPPTPT, where this shoulder and red-shift are absent in $o \mathrm{DCB}$ at $140{ }^{\circ} \mathrm{C}$. The values extracted from the GPC trace are therefore likely overestimated for 1B-DT-PDPPTPT. Nevertheless, the branched polymer showed both lower number average $\left(M_{\mathrm{n}}\right)$ and lower peak $\left(M_{\mathrm{p}}\right)$ molecular weights of 31.4 and $43.1 \mathrm{kDa}$ respectively, compared to the linear polymer (45.4 and $85.0 \mathrm{kDa}$ ) (See ESI, $†$ Table S1). A further problem with this analysis is that GPC estimates the molecular weight based on hydrodynamic volume, and the exact impact of branching in stiff polymers on this volume is not well known. As branching diminishes the hydrodynamic volume, the molecular weight of the branched polymer could be underestimated.

Photovoltaic cells of both polymers were made using [6,6]phenyl- $\mathrm{C}_{71}$-buteric acid methyl ester ([70]PCBM) as an acceptor, sandwiching the active layer between ITO/PEDOT:PSS and $\mathrm{LiF} / \mathrm{Al}$ electrodes. The active layers were optimized in terms of thickness, polymer-fullerene ratio, and solvent composition of the casting solution. It was found that the optimal conditions for 1B-DT-PDPPTPT were similar to the previously ${ }^{5}$ optimized conditions for DT-PDPPTPT, using a $1: 2$ polymer to [70]PCBM weight ratio dissolved in a mixture of chloroform with $3 \%$ of 1,8-diiodooctane (DIO), while the best cell of DT-PDPPTPT in this study was made using $6 \%$ of DIO. The photovoltaic performance is summarized in Table 1 and Fig. 2. The open circuit voltage $\left(V_{\text {oc }}\right)$ was found to be very similar for both materials, as was expected from the similar backbone. The fill factor (FF) does not show a big difference either, but an important difference was found in the short circuit current density $\left(J_{\mathrm{sc}}\right)$. A dramatic increase of from 8.7 to $12.5 \mathrm{~mA} \mathrm{~cm}{ }^{-2}$ resulted in an improved power conversion efficiency (PCE) of 6.3\% for 1B-DT-PDPPTPT compared to $4.4 \%$ for DT-PDPPTPT for the best devices. As can be seen Fig. $1 \mathrm{~b}$ the branched polymer shows a higher response in the spectrally resolved external quantum efficiency (EQE) over the whole absorption range of the device. Although there is a small difference in absorption, most of this increase is caused by a change in internal quantum efficiency (IQE) (Fig. S1, ESI $\dagger$ ).

To investigate the morphology, transmission electron microscopy (TEM) was performed on the active layers of the cells (Fig. 3). Both blends show a fibre-like morphology, as was seen
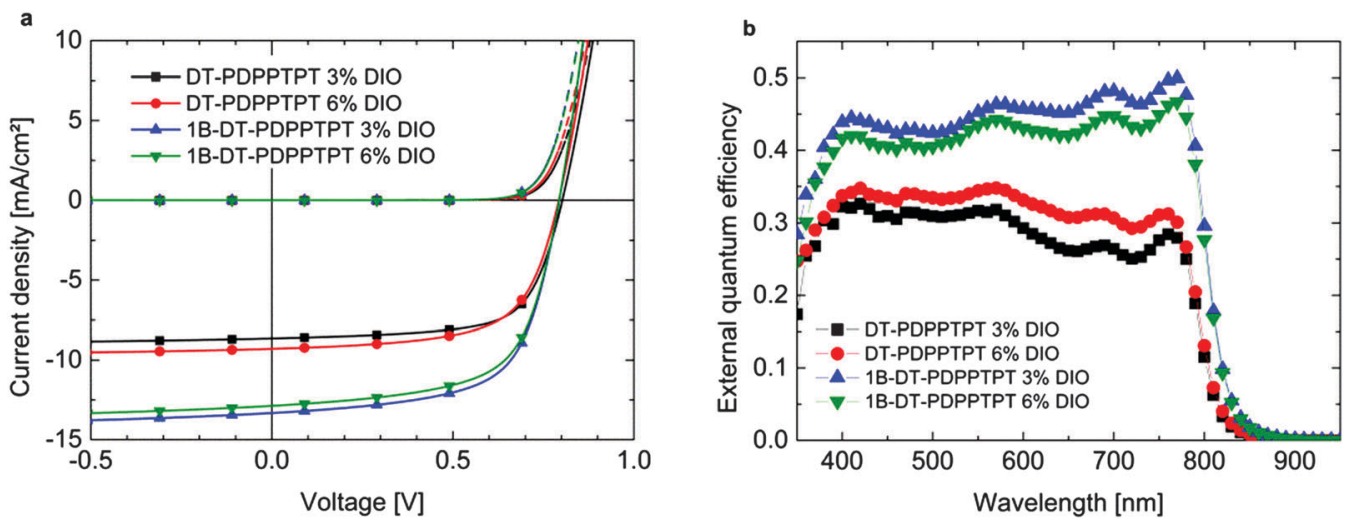

Fig. 2 (a) Current density-voltage ( $J-V$ and (b) external quantum efficiency (EQE) of DT-PDPPTPT:[70]PCBM and 1B-DT-PDPPTPT:[70]PCBM solar cells processed from chloroform using $3 \%$ and $6 \%$ of DIO as co-solvent. 

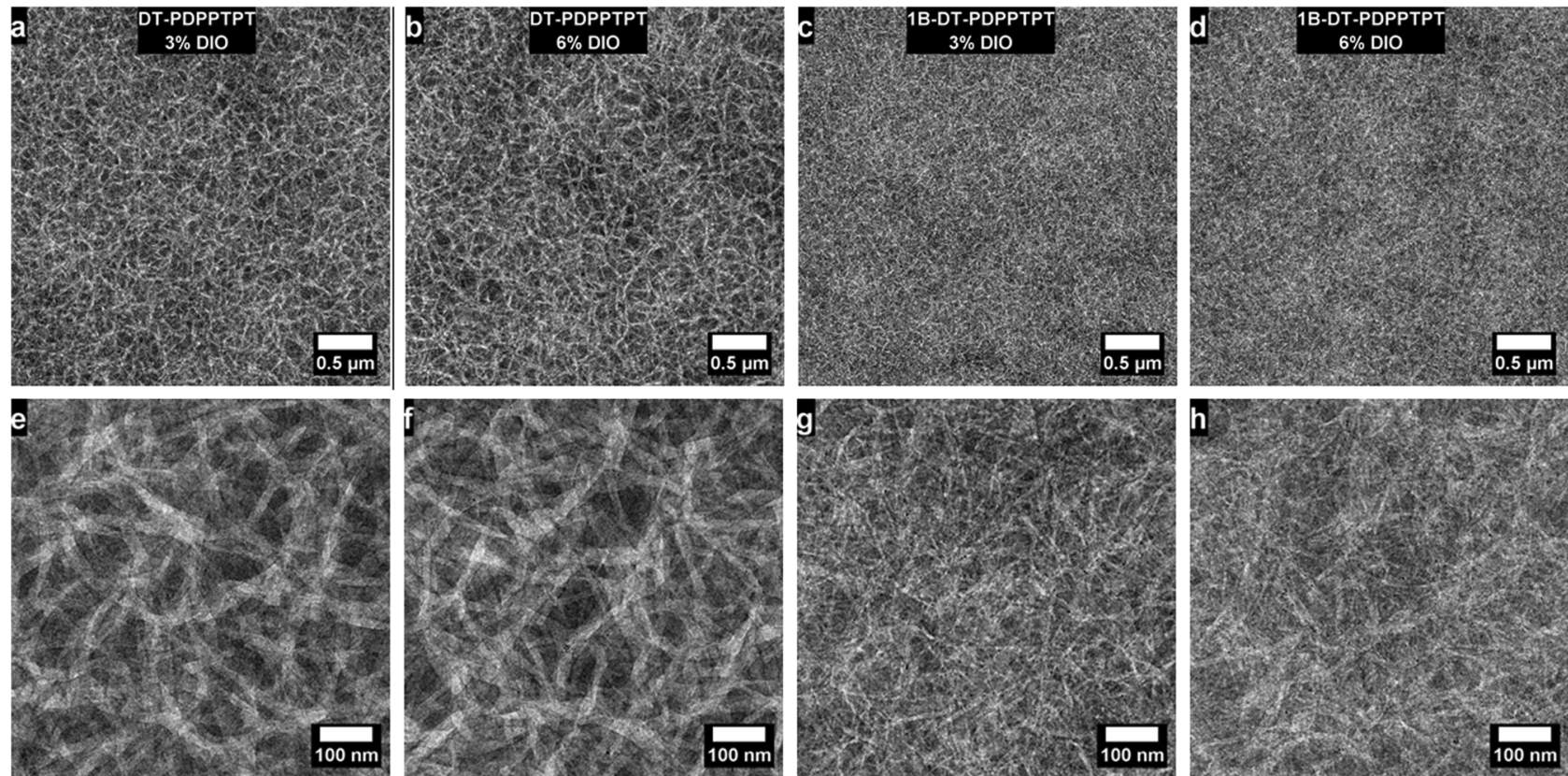

Fig. 3 TEM images of the active layers of DT-PDPPTPT processed with 3\% DIO (a and e), DT-PDPPTPT with 6\% DIO (b and f), 1B-DT-PDPPTPT with $3 \% \mathrm{DIO}(\mathrm{c}$ and $\mathrm{g}$ ) and 1B-DT-PDPPTPT with 6\% DIO ( $\mathrm{d}$ and $\mathrm{h}$ ).

earlier in many DPP-containing polymers. ${ }^{5,6,15}$ The fringes observed in the TEM of these fibres at high magnification indicate that they consist of relatively pure semi-crystalline polymer. There is however a large difference between the fibre width in both blends. The branching units in 1B-DT-PDPPTPT significantly reduce the fibre width compared to DT-PDPPTPT, leading to a more intimately mixed morphology, explaining the increased EQE and gain in efficiency, as charge generation is more efficient. It has been shown that increasing the molecular weight in DPP-polymers leads to more finely dispersed fibremorphologies, ${ }^{15}$ however, in this case the GPC results indicate the opposite to be true. Even when taking into account errors in this measurement induced by the branched nature of the polymer, it is unlikely that the molecular weight of 1B-DTPDPPTPT is dramatically higher than that of DT-PDPPTPT, and the difference in morphology in both blends is greater than any change we have seen in past due to a small change in molecular weight. Therefore, we hypothesize that another effect, caused by the branching, is at play here.

To gain more insight in the morphology formation, in situ light scattering experiments were performed during spin coating of the layer. ${ }^{22}$ In this experiment, the polymer-fullerene solutions were spin coated onto a silicon wafer covered with PEDOT:PSS. The thickness of the liquid layer during spin coating was monitored by recording the interference of a specular reflected laser beam, while a photodetector placed to the side of this set-up recorded simultaneously the light scattered from the solution. Given the dimensions of the polymer fibres, we conjecture that an increase in scattering signal indicates fibre formation. This experiment was performed on active layer blends of both materials, using both $3 \%$ and $6 \%$ of DIO as co-solvent concentration. In all experiments, a fast ( $<1$ s) drying regime (Fig. 4 and Fig. S2, ESI $\dagger$ ) and a slow (>1 h) drying regime (Fig. S3, ESI $\dagger$ ) can be distinguished, which we attribute to the evaporation of chloroform in the first stage of drying, followed by a long drying period for DIO. ${ }^{15}$ To be able to directly compare the experiments, the curves were synchronized to the point where all chloroform is evaporated (Fig. 4a, for more details see ESI $\dagger$ ). In Fig. 4b, it can be seen that the branched polymer shows an onset of scattering

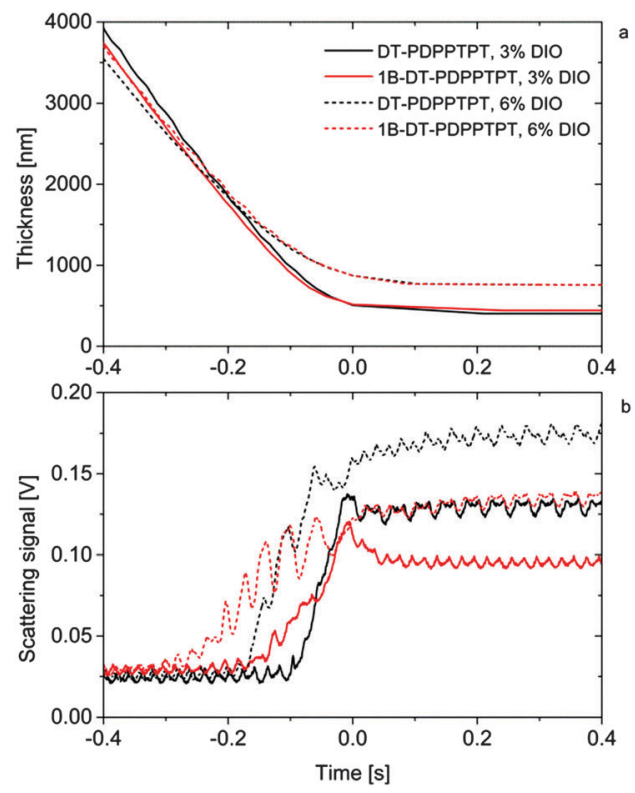

Fig. 4 Drying curve (a) and scattering signal of laser light (b) on the mixture of polymer, fullerene, solvent and co-solvent during spin coating versus time. The signals of different experiments are manually synchronized at the point of drying for the chloroform. 
before the linear polymer, meaning that fibre formation starts earlier, at lower concentration, when the same solvent mixture is used. Using the final thickness of the layers, the polymer content by volume at the onset of scattering was calculated to be $9.3 \%$ and $7.7 \%$ for the linear and branched polymer respectively when $3 \%$ DIO was used, and $6.2 \%$ and $4.0 \%$ for $6 \%$ DIO. However, the polymer concentration at the onset of scattering does not seem to determine the fibre width, as can be seen when comparing the linear polymer spin coated from $6 \%$ DIO in chloroform with the branched polymer from $3 \%$ DIO in chloroform. In these cases, the linear polymer has an onset of scattering at a higher solvent content than the branched polymer, yet the former has a larger fibre width (Fig. 3).

We have recently shown that the width of the semicrystalline polymer fibres in bulk heterojunction blends is not the result of a frozen dynamical state but determined by nucleation, which is governed by the solubility of the polymer and related to the side chains, the molecular weight and the nature of the solvent/ co-solvent system. ${ }^{15}$ Also the present results can be interpreted in the context of this nucleation-and-growth model. ${ }^{15}$ Classic nucleation theory states that that a free energy barrier must be overcome to form a nucleus of critical size, which can subsequently grow. The higher this barrier is, the lower the number of nuclei will be, and the bigger they will be. A large barrier for nucleation can therefore result in wide fibres either because a small number of nuclei grow into large aggregates (when there is a long growth period) or because the nuclei are large and the fibre size is determined by this (when there is a very limited growth time). For DT-PDPPTPT a large free energy barrier can be expected as it has good solubility owing to the lengthy DT chain resulting in relatively wide fibres, which is consistent with our observations. According to this theory, the branched polymer must have a lower energy barrier as the fibres are found to be thinner. An increased tendency of aggregation is consistent with the observations made in the GPC analysis and the decreased solubility observed in the light scattering experiments, lending credence to this theory. The correlation between polymer solubility and fibre width has been pointed out before. ${ }^{23,24}$ The origin of the lowered barrier for nucleation is not clear at this point, although one might speculate that the branching causes a decreased number of degrees of freedom of the polymer in solution, leading to a lower entropic penalty for nucleation.

In conclusion, introducing only $1 \%$ of branching in DTPDPPTPT has a significant effect on the photovoltaic performance and results in a PCE of $6.3 \%$, compared to $4.4 \%$ for the linear polymer due to an increase in photocurrent. This increase is explained by a reduction of the polymer fibre width in the active layer. In situ scattering experiments revealed an onset of fibre formation during spin coating at lower concentration for the branched material, which was explained in terms of a lowered solubility. The increased tendency of aggregation is consistent with a lower barrier for nucleation, leading to thinner fibres through the theory of nucleation-and-growth. We propose that this decrease in the energetic barrier for nucleation finds its origin in entropic effects caused by the branched nature of the polymer. The results therefore demonstrate that introducing branching can be an effective tool to increase photovoltaic efficiency.

We thank Ralf Bovee for GPC analysis. G. H. L. Heintges acknowledges the Agency for Innovation by Science and Technology in Flanders (IWT). This research forms part of the research program of the Dutch Polymer Institute (DPI), project \#734 and received funding from the Ministry of Education, Culture and Science (Gravity program 024.001.035).

\section{Notes and references}

1 Z. He, B. Xiao, F. Liu, H. Wu, Y. Yang, S. Xiao, C. Wang, T. P. Russell and Y. Cao, Nat. Photonics, 2015, 9, 174-179.

2 Y. Liu, J. Zhao, Z. Li, C. Mu, W. Ma, H. Hu, K. Jiang, H. Lin, H. Ade and H. Yan, Nat. Commun., 2015, 5, 5293.

3 S.-H. Lia, H.-J. Jhuo, P.-N. Yeh, Y.-S. Cheng, Y.-L. Li, Y.-H. Lee, S. Sharma and S.-A. Chen, Sci. Rep., 2014, 4, 6813.

4 J.-D. Chen, C. Cui, Y.-Q. Li, L. Zhou, Q.-D. Ou, C. Li, Y. Li and J.-X. Tang, Adv. Mater., 2015, 27, 1035-1041.

5 W. Li, K. H. Hendriks, A. Furlan, W. S. C. Roelofs, M. M. Wienk and R. A. J. Janssen, J. Am. Chem. Soc., 2013, 135, 18942-18948.

6 K. H. Hendriks, G. H. L. Heintges, V. S. Gevaerts, M. M. Wienk and R. A. J. Janssen, Angew. Chem., Int. Ed., 2013, 52, 8341-8344.

7 L. Dou, H.-H. Chang, J. Gao, C.-C. Chen, J. Jou and Y. Yang, Adv. Mater., 2013, 25, 825-831.

8 Y. Li, P. Sonar, L. Murphy and W. Hong, Energy Environ. Sci., 2013, 6, 1684-1710.

9 J.-R. Pouliot, B. Sun, M. Leduc, A. Najari, Y. Li and M. Leclerc, Polym. Chem., 2015, 6, 278-282.

10 C. Duan, F. Huang and Y. Cao, J. Mater. Chem., 2012, 22, 10416-10434. 11 C. Cabanetos, A. El Labban, J. A. Bartelt, J. D. Douglas, W. R. Mateker, J. M. J. Fréchet, M. D. McGehee and P. M. Beaujuge, J. Am. Chem. Soc., 2013, 135, 4656-4659.

12 W.-H. Chang, J. Gao, L. Dou, C.-C. Chen, Y. Liu and Y. Yang, Adv. Energy Mater., 2014, 4, 1300864.

13 J. K. Lee, W. L. Ma, C. J. Brabec, J. Yuen, J. S. Moon, J. Y. Kim, K. Lee, G. C. Bazan and A. J. Heeger, J. Am. Chem. Soc., 2008, 130, 3619-3623.

14 H.-C. Liao, C.-C. Ho, C.-Y. Chang, M.-H. Jao, S. B. Darling and W.-F. Su, Mater. Today, 2013, 16, 326-336.

15 J. J. van Franeker, G. H. L. Heintges, C. Schaefer, G. Portale, W. Li, M. M. Wienk, P. van der Schoot and R. A. J. Janssen, J. Am. Chem. Soc., 2015, 137, 11783-11794.

16 E. Zhou, J. Cong, K. Hashimoto and K. Tajima, Energy Environ. Sci., 2012, 5, 9756-9759.

17 Z. Gu, P. Tang, B. Zhao, H. Luo, X. Guo, H. Chen, G. Yu, X. Liu, P. Shen and S. Tan, Macromolecules, 2012, 45, 2359-2366.

18 J. Qiao, C. Yang, Q. He, F. Bai and Y. Li, J. Appl. Polym. Sci., 2004, 92, 1459-1466.

19 C. Q. Ma, E. Mena-Osteritz, T. Debaerdemaeker, M. M. Wienk, R. A. Janssen and P. Bäuerle, Angew. Chem., Int. Ed., 2007, 46, 1679-1683.

20 C.-Q. Ma, M. Fonrodona, M. C. Schikora, M. M. Wienk, R. A. J. Janssen and P. Bäuerle, Adv. Funct. Mater., 2008, 18, 3323-3331.

21 H. S. Mangold, T. V Richter, S. Link, U. Würfel and S. Ludwigs, J. Phys. Chem. B, 2012, 116, 154-159.

22 J. J. van Franeker, M. Turbiez, W. Li, M. M. Wienk and R. A. J. Janssen, Nat. Commun., 2015, 6, 6229.

23 W. Li, K. H. Hendriks, A. Furlan, W. S. C. Roelofs, S. C. J. Meskers, M. M. Wienk and R. A. J. Janssen, Adv. Mater., 2014, 26, 1565-1570.

24 X. Cao, M. Li, J. Liu, H. Wang, K. Zhou and Y. Han, Org. Electron., 2015, 24, 280-287. 